\title{
Assessment of the Asian Neurogastroenterology and Motility Association Chronic Constipation Criteria: An Asian Multicenter Cross-sectional Study
}

\begin{abstract}
Kok-Ann Gwee, ${ }^{1 *}$ Paul Bergmans, ${ }^{2}$ JinYong Kim, ${ }^{3}$ Bogdana Coudsy, ${ }^{4}$ Angelia Sim, ${ }^{5}$ Minhu Chen, ${ }^{6}$ Lin Lin, ${ }^{7}$ Xiaohua Hou, ${ }^{8}$ Huahong Wang, ${ }^{9}$ Khean-Lee Goh, ${ }^{10}$ John A Pangilinan, ${ }^{11}$ Nayoung Kim, ${ }^{12}$ and Stanislas Bruley des Varannes ${ }^{13}$

${ }^{1}$ Department of Medicine, Yong Loo Lin School of Medicine, National University of Singapore, Singapore; ${ }^{2}$ Biometrics Department, Janssen Cilag Benelux, Tilburg, The Netherlands; ${ }^{3}$ Regional Medical Affairs, Janssen Asia-Pacific, Singapore; ${ }^{4} J a n s s e n$ EMEA, Issy-les-Moulineaux, France; ${ }^{5}$ Global Clinical Operations, Janssen, Selangor, Malaysia; ${ }^{6}$ Department of Gastroenterology and Hepatology, The First Affiliated Hospital of Sun Yat-Sen University, Guangzhou, China; 'Department of Gastroenterology, The First Affiliated Hospital of Nanjing Medical University People's Hospital of Jiangsu Province, China; ${ }^{8}$ Department of Gastroenterology and Hepatology, Union Hospital of Tongji Medical College, Huazhong University of Science and Technology, Wuhan, Hubei, China; ${ }^{9}$ Gastrointestinal Department, Peking University First Hospital, Beijing; China; ${ }^{10}$ University of Malaya, Kuala Lumpur, Malaysia; ${ }^{11}$ Department of Internal Medicine Section of Gastroenterology, St Luke's Medical Center, Institute of Digestive and Liver Diseases, Quezon, The Philippines; ${ }^{12}$ Department of Internal Medicine, Seoul National University Bundang Hospital, Seongnam, Korea; and ${ }^{13}$ Institut des Maladies de l'Appareil Digestif, University Hospital of Nantes, Nantes, France
\end{abstract}

\section{Background/Aims}

There is a need for a simple and practical tool adapted for the diagnosis of chronic constipation (CC) in the Asian population. This study compared the Asian Neurogastroenterology and Motility Association (ANMA) CC tool and Rome III criteria for the diagnosis of CC in Asian subjects.

\section{Methods}

This multicenter, cross-sectional study included subjects presenting at outpatient gastrointestinal clinics across Asia. Subjects with CC alert symptoms completed a combination Diagnosis Questionnaire to obtain a diagnosis based on 4 different diagnostic methods: self-defined, investigator's judgment, ANMA CC tool, and Rome III criteria. The primary endpoint was the level of agreement/ disagreement between the ANMA CC diagnostic tool and Rome III criteria for the diagnosis of CC.

\section{Results}

The primary analysis comprised of 449 subjects, 414 of whom had a positive diagnosis according to the ANMA CC tool. Rome III positive/ANMA positive and Rome III negative/ANMA negative diagnoses were reported in $76.8 \%$ and $7.8 \%$ of subjects, respectively, resulting in an overall percentage agreement of $84.6 \%$ between the 2 diagnostic methods. The overall percentage disagreement between these 2 diagnostic methods was $15.4 \%$. A higher level of agreement was seen between the ANMA CC tool and self-defined (374 subjects [90.3\%]) or investigator's judgment criteria (388 subjects [93.7\%]) compared with the Rome III criteria.

\section{Conclusion}

This study demonstrates that the ANMA CC tool can be a useful for Asian patients with CC.

(J Neurogastroenterol Motil 2017;23:262-272)

Key Words

Asia; Constipation; Cross-sectional studies; Humans; Practice guidelines as topic/standards

Received: June 13, 2016 Revised: September 5, 2016 Accepted: September 16, 2016

(.) This is an Open Access article distributed under the terms of the Creative Commons Attribution Non-Commercial License (http://creativecommons. org/licenses/by-nc/4.0) which permits unrestricted non-commercial use, distribution, and reproduction in any medium, provided the original work is properly cited.

*Correspondence: Kok-Ann Gwee, FRCP, PhD

Stomach Liver and Bowel Centre, Gleneagles Hospital, Annexe Block 05-37, 6A Napier Road, Singapore 258500, Singapore Tel: +65-6474-6848, Fax: +65-6470-5616, E-mail: slbclinic@gmail.com 


\section{Introduction}

Chronic constipation (CC) is a common disorder that can have a significant impact on the quality of life (QoL). The estimated prevalence of self-defined (SD) constipation in Asia (South Korea, China, and Indonesia) is $15-23 \%$ in women and approximately $11 \%$ in men. ${ }^{1}$ However, difficulty still exists in recognizing and establishing a diagnosis of $\mathrm{CC}$. In a study of the Rome I diagnostic criteria, $66 \%$ of subjects with self-perceived CC and $71 \%$ of subjects with slow colonic transit times failed to meet the criteria. ${ }^{2}$ Furthermore, up to $65 \%$ of patients who met the criteria reported that they did not feel constipated. ${ }^{2}$

The differing perceptions among physicians and patients regarding what constitutes constipation is another reason why the diagnosis of $\mathrm{CC}$ is such a challenge. ${ }^{3}$ Indeed, one study has reported that $27 \%$ of patients regarded a defecation frequency of once every 2 days as constipation, whereas the majority (73\%) of physicians considered defecation once every 3 days as constipation. ${ }^{4}$ More recently, in a study of 26 patients with irritable bowel syndrome (IBS) from South Korea who considered that they were passing hard stools, only 7 met the definition of hard stool by the Rome III criteria (Bristol stool form scale type 1 or type 2$)^{5}$

These findings highlighted the need for a simple and practical tool adapted for the diagnosis of $\mathrm{CC}$ in the Asian population. As a result, the ANMA proposed a practical approach to the diagnosis of CC, which they called the ANMA CC tool. ${ }^{6}$ Diagnosis of CC using this tool adopts a two-step approach. In the first step, physicians are alerted to the possibility of $\mathrm{CC}$ by the presence of "alert symptoms" frequently associated with CC; these comprise of bloating, fullness, difficulty to pass stools, and the need for laxatives, as well as the usual constipation symptoms. ${ }^{6}$ In the presence of "alert symptoms," physicians ask the patient if they have experienced any of the 6 constipation symptoms in the Rome III criteria list on a regular basis; these consist of $<3$ bowel movements per week, straining, the presence of lumpy or hard stools, the sensation of anorectal obstruction, the sensation of incomplete defecation, and manual maneuvers.

The aim of this study was to assess the agreement/disagreement between the ANMA CC tool and the Rome III criteria for the diagnosis of $\mathrm{CC}$ in Asian subjects consulting in gastroenterology clinics.

\section{Materials and Methods}

\section{Study Design}

The Constipation Symptoms Observational Study was a multicenter, cross-sectional, epidemiologic study that included primary and secondary care subjects presenting at 17 outpatient gastrointestinal clinics across Asia (China, South Korea, Malaysia, the Philippines, and Singapore). The study protocol was approved at each institution by an independent ethics committee or institutional review board, and was conducted in accordance with the Helsinki Declaration (ClinicalTrials.gov identifier: NCT01880294).

The study comprised of 2 stages. During Stage 1, all Asian subjects presenting with any condition at the participating clinics during investigator-chosen consecutive days (within a 3-month period) completed a screening worksheet incorporating the ANMA $\mathrm{CC}$ tool to determine the presence of $\mathrm{CC}$ alert symptoms. A maximum of 55 consecutive subjects were enrolled at each site, regardless of the number of consultation days chosen. At Stage 1, a question on the ANMA CC Tool worksheet was: "Do you think you have chronic constipation?" Patients who answered this question positively were considered to have a positive SD diagnosis of CC. Stage 2 was divided into 2 sub-stages. During Stage 2A, eligible subjects completed a combination Diagnosis Questionnaire (Appendix) to obtain a diagnosis of CC based on investigator's judgment (INV), ANMA CC tool, and Rome III criteria. The Diagnosis Questionnaire also collected information on previous complementary examinations, Patient Assessment of Constipation Symptom (PAC-SYM) score and demographic details. Subjects with a positive diagnosis of CC based on the ANMA CC tool proceeded to Stage $2 \mathrm{~B}$, which involved the completion of a Documentation Questionnaire to collect additional information on the subject's demographic details, medical history, history of CC, current symptoms of $\mathrm{CC}$, comorbidities, and past and current medications for CC. Subjects who entered Stage 2B also completed the Patient Assessment of Constipation-Quality of Life (PAC-QOL) questionnaire.

\section{Study Population}

Eligible subjects were male or female Asian subjects $(\geq 18$ years of age) with $\geq 1$ of the CC alert symptoms listed in the worksheet that was present for $\geq 3$ months. Exclusion criteria included $\mathrm{CC}$ that was drug-induced or due to secondary causes, surgical obstruction, megacolon/megarectum, a diagnosis of pseudo- 
obstruction or organic disorders of the large bowel. Female subjects who were pregnant were also excluded.

\section{Evaluations}

The primary endpoints were the proportion of subjects for whom the $\mathrm{CC}$ diagnosis according to the ANMA CC tool agreed with the diagnosis according to the Rome III criteria, and the proportion of subjects for whom the $\mathrm{CC}$ diagnosis according to the ANMA CC tool and the Rome III criteria did not agree.

Secondary endpoints were: (1) point prevalence rates for all diagnostic methods using the number of screened subjects as the denominator, (2) the concordance of the four diagnostic methods and agreement or disagreement on item levels of the different questionnaires, (3) analysis of the use of CC therapy, (4) analysis of the difference between investigator's and subject's judgment about CC diagnosis with regard to current symptoms and comorbidities, (5) PAC-SYM, ${ }^{7}$ and (6) PAC-QOL. ${ }^{8}$

\section{Statistical Methods}

No formal sample size calculations were performed because of the exploratory nature of the study. It was anticipated that the screening of approximately 2000 consecutive subjects would be required to yield an estimated 400 subjects with suspected CC. The estimated sample size of 400 produced a two-sided $95 \%$ confidence interval $(\mathrm{CI})$ with a width equal to 0.058 when the sample proportion was 0.1 . For a sample proportion of 0.5 , the width of the $\mathrm{CI}$ increased to 0.098 (exact confidence limit according Wilson).

The screening population was defined as all subjects who entered Stage 1. The screened positive population comprised of all subjects who scored positively for $\mathrm{CC}$ alert symptoms on the ANMA worksheet and provided informed consent. The positive ANMA diagnosis population was defined as all subjects from the screened positive population who had a positive diagnosis of $\mathrm{CC}$ according to the ANMA CC tool.

The descriptive analysis of nominal/ordinal data comprised of tabulation of frequency and percentages, continuous data of the mean, standard deviation, median, extreme values, and two-sided 95\% CIs. Descriptive analysis of prior CC therapy for all subjects was also provided. There was no adjustment for multiplicity.

\section{Results}

A total of 4570 subjects entered Stage 1 of which 457 had $\geq 1$ of the CC alert symptoms (Fig. 1). Eight subjects with secondary

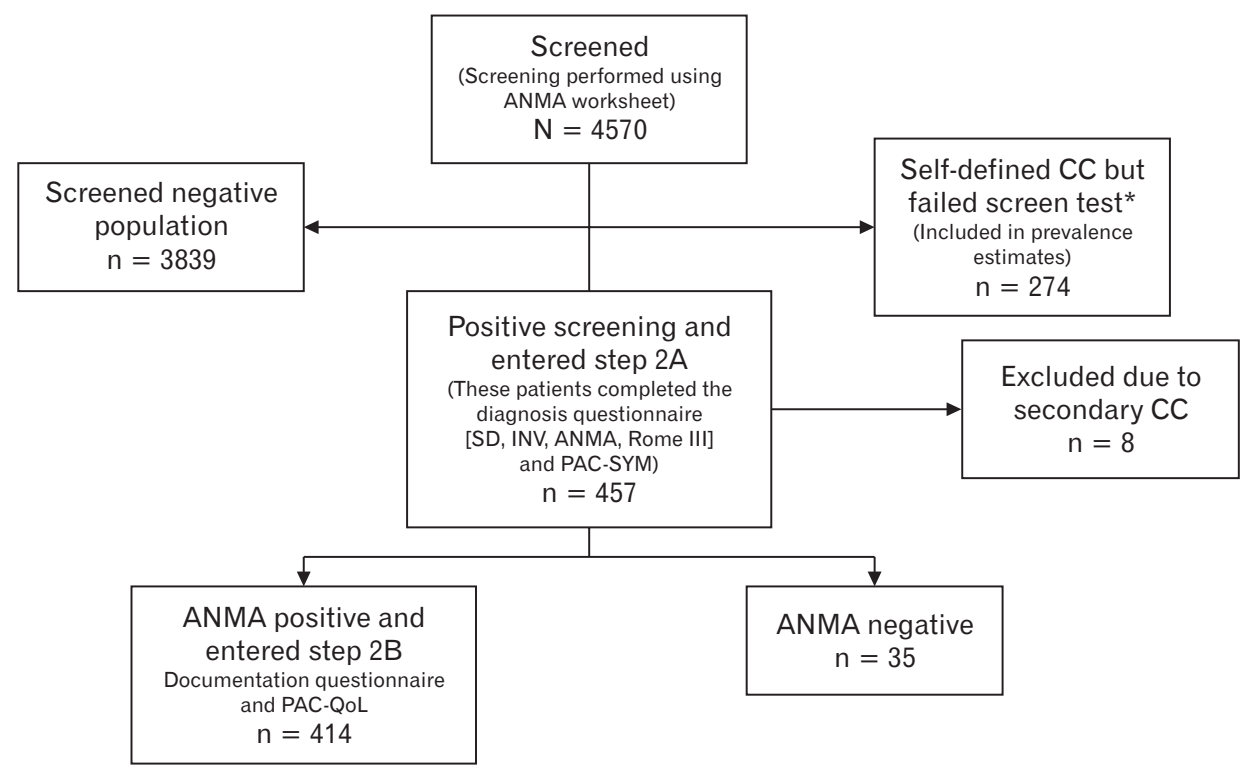

Figure 1. Patient disposition. Flow scheme of the 2-step design of this study. The initial stage was a screening step which included 4570 patients. Of these, 457 participants entered Stage 2 based on the Asian Neurogastroenterology and Motility Association (ANMA) worksheet which identified the presence of chronic constipation (CC) alert symptoms. Stage 2 was divided into Stage 2A and Stage 2B. Only patients who were positive with the ANMA CC tool $(n=414)$ proceeded to Stage 2B. SD, self-defined; INV, investigator-defined; PAC-SYM, Patient Assessment of Constipation Symptom Score; PAC-QOL, Patient Assessment of Constipation-Quality of Life. *These patients considered themselves to have CC but did not report any alert symptoms within the screen test. They were excluded from subsequent stages of the study but were included in estimates of prevalence. 
or drug-induced $\mathrm{CC}$ were excluded. As a result, the primary analysis comprised of 449 subjects (ie, the screened positive population). Overall, 414 subjects had a positive $\mathrm{CC}$ diagnosis according to the ANMA CC tool and completed Stage 2B of the study (ie, the positive ANMA diagnosis population).

The majority of subjects were female (304 subjects [67.7\%]) and Chinese (294 subjects [65.5\%]) (Table 1). The overall mean (standard deviation) age and weight were 51.4 (16.4) years and 60.1 (9.6) kg, respectively.

\section{Primary Analysis}

The primary analysis was conducted on the screened positive population $(\mathrm{n}=449)$. Twelve subjects with a Rome III positive $(+) /$ ANMA negative (-) diagnosis were excluded because of anomalies in the timescale of symptom reporting, resulting in these patients failing to meet ANMA criteria, even though they were Rome III+. Overall, the proportions of subjects with Rome III+/ANMA+ and Rome III-/ANMA- diagnoses were $76.8 \%$ and $7.8 \%$, respectively. Therefore, the overall agreement between the ANMA CC tool and Rome III criteria was $84.6 \%$ (380/449 subjects). The overall disagreement between these 2 methods (ie, a Rome III-/ ANMA + diagnosis) was $15.4 \%$ (69/449 subjects). Sensitivity analyses including the 12 Rome III+/ANMA- subjects who were excluded from the primary analysis did not show any relevant differences compared with the primary analysis (data not shown).

A total of 318 subjects had a positive diagnosis of CC using all 4 methods. A positive diagnosis using 3 out of the 4 methods was reported in 63 subjects, which included: 40 subjects who were

Table 1. Subject Demographics (Screened Positive Population, $\mathrm{N}=$ 449)

\begin{tabular}{lc}
\hline & All subjects \\
\hline Mean age $(\mathrm{yr}[\mathrm{SD}])$ & $51.4(16.4)$ \\
Gender $(\mathrm{n}[\%])$ & $304(67.7)$ \\
$\quad$ Female & $145(32.3)$ \\
$\quad$ Male & $22.8(3.17)$ \\
Mean BMI $\left(\mathrm{kg} / \mathrm{m}^{2}[\mathrm{SD}]\right)$ & \\
Ethnicity $(\mathrm{n}[\%])$ & $294(65.5)$ \\
Chinese & $84(18.7)$ \\
South Korean & $46(10.2)$ \\
Filipino & $11(2.4)$ \\
Malaysian & $7(1.6)$ \\
Indian & $7(1.6)$ \\
${ }^{a}$ Other & \\
\hline
\end{tabular}

${ }^{a}$ Other ethnicities included Indonesian, Thai, Filipino-Chinese and Sabahan. BMI, body mass index.
$\mathrm{ANMA}+, \mathrm{SD}+, \mathrm{INV}+$, and Rome III-; 14 subjects who were $\mathrm{ANMA}+$, Rome III+, INV+, and SD-; and nine subjects who were $\mathrm{ANMA}+$, Rome III+, SD+, and INV- (Table 2). Positive diagnoses of $\mathrm{CC}$ according to only 1 or 2 of the 4 methods were reported in 44 subjects. There were 24 subjects who passed the screening tool but who had a negative diagnosis on all 4 diagnostic methods.

\section{Prevalence of Chronic Constipation}

In the total screening population $(\mathrm{n}=4570)$, the prevalence of CC was: SD, $14.4 \%$; ANMA CC tool, $9.1 \%$; INV, $8.6 \%$; and Rome III, $7.5 \%$ (Table 3 ). CC prevalence rates were higher in females than in males according to all diagnostic methods. In general, prevalence according to SD criteria was higher than the other 3 diagnostic methods due to the identification of SD CC within Stage 1, where 274 patients identified themselves as having SD CC but they did not report any CC alert symptom, and therefore did not progress to Stage 2A. Overall, within the screened population, 656 patients reported SD CC: 382 also reported $\geq 1$ alert symptom and progressed to Stage $2 \mathrm{~A}$, and the remaining 274 reported no alert symptoms and were excluded.

\section{Concordance}

A high level of agreement was seen between the diagnosis of $\mathrm{CC}$ according to the ANMA CC tool and SD or INV criteria (Fig. 2A). Most subjects who had a positive diagnosis of CC using the ANMA CC tool also had positive diagnoses according to SD (374 subjects [90.3\%]) and INV (388 subjects [93.7\%]) criteria. Similarly, the majority of the 35 ANMA- subjects were also SD- (27 subjects [77.1\%]) and INV- (28 subjects [80.0\%]) (Fig. 2A). As a result, the overall concordance was $89.3 \%$ between the ANMA $\mathrm{CC}$ tool and SD criteria, and $92.7 \%$ between the ANMA CC tool and INV criteria (Fig. 3A).

Table 2. Diagnosis of Chronic Constipation According to Various Diagnostic Methods $(\mathrm{N}=449)$

\begin{tabular}{lcccc}
\hline & \multicolumn{4}{c}{ Patients (n [\%]) } \\
\cline { 2 - 5 } & $318(70.8)$ & $40(8.9)$ & $9(2.0)$ & $14(3.1)$ \\
\hline CC self-defined & + & + & + & - \\
CC investigator & + & + & - & + \\
ANMA CC tool & + & + & + & + \\
CC Rome III criteria & + & - & + & + \\
\hline
\end{tabular}

ANMA, Asian Neurogastroenterology and Motility Association; CC, chronic constipation. 
Table 3. Prevalence (\%) of Chronic Constipation According to Different Diagnostic Methods (Screening Population, $\mathrm{N}=4570$ )

\begin{tabular}{lcccc}
\hline & ANMA CC tool & CC Rome III criteria & CC investigator-defined & CC self-defined \\
\hline Total $(\mathrm{N}=4570)$ & 9.1 & 7.5 & 8.6 & 14.4 \\
Gender & & & 5.8 & 10.9 \\
Male $(\mathrm{n}=1993)$ & 6.1 & 9.6 & 10.9 & 17.0 \\
Female $(\mathrm{n}=2577)$ & 11.3 & & & 6.8 \\
Country & & 5.9 & 16.0 & 14.3 \\
China $(\mathrm{n}=3225)$ & 6.9 & 14.2 & 6.9 & 21.3 \\
South Korea $(\mathrm{n}=450)$ & 18.4 & 6.3 & 17.7 & 8.1 \\
Malaysia $(\mathrm{n}=540)$ & 7.2 & 15.5 & 21.4 & 17.7 \\
Philippines $(\mathrm{n}=271)$ & 18.1 & 19.0 & 9.5 \\
Singapore $(\mathrm{n}=84)$ & 23.8 & & \\
\hline
\end{tabular}

ANMA, Asian Neurogastroenterology and Motility Association; CC, chronic constipation.

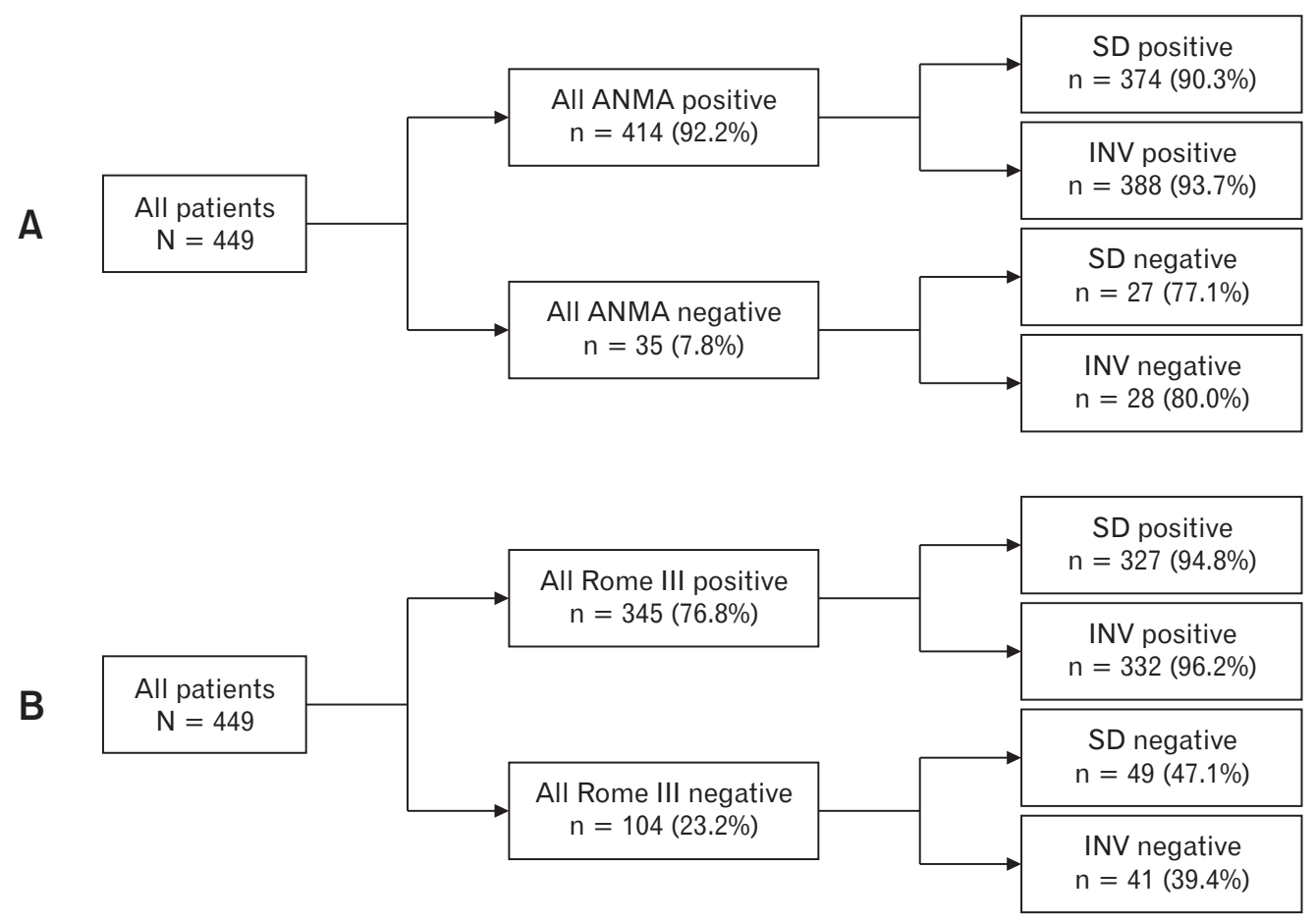

Figure 2. Concordance between Asian Neurogastroenterology and Motility Association (ANMA) chronic constipation (CC) tool or Rome III criteria and self-defined (SD) or investigator-defined (INV) chronic constipation. (A) Of the 449 patients evaluated, 414 were ANMA CC-positive. Most ANMA-positive individuals $(\mathrm{n}=414)$ were also SD-positive $(\mathrm{n}=374)$ and INV-positive $(\mathrm{n}=388)$. Similarly, the majority of individuals who were defined as ANMA-negative were also SD-negative and INV-negative. (B) Overall 345/449 patients (76.8\%) were Rome III-positive. A high proportion of Rome III-positive patients were also positive according to SD-positive and INV-positive; but $>50 \%$ of patients who were Rome III-negative were either SD-negative or INV-negative.

The majority of the 345 subjects who had a positive diagnosis of CC according to the Rome III criteria also had positive diagnoses according to SD (327 subjects [95.0\%]) and INV (332 subjects [96.2\%]) criteria (Fig. 2B). Overall concordance was $83.7 \%$ between the Rome III and SD criteria, and $83.1 \%$ between the Rome III and INV criteria (Fig. 3A). In contrast to the findings in ANMA- subjects, < 50\% of the 104 Rome III- subjects were SD- (49 subjects [47.1\%]) or INV- (41 subjects [39.4\%]) (Fig. 

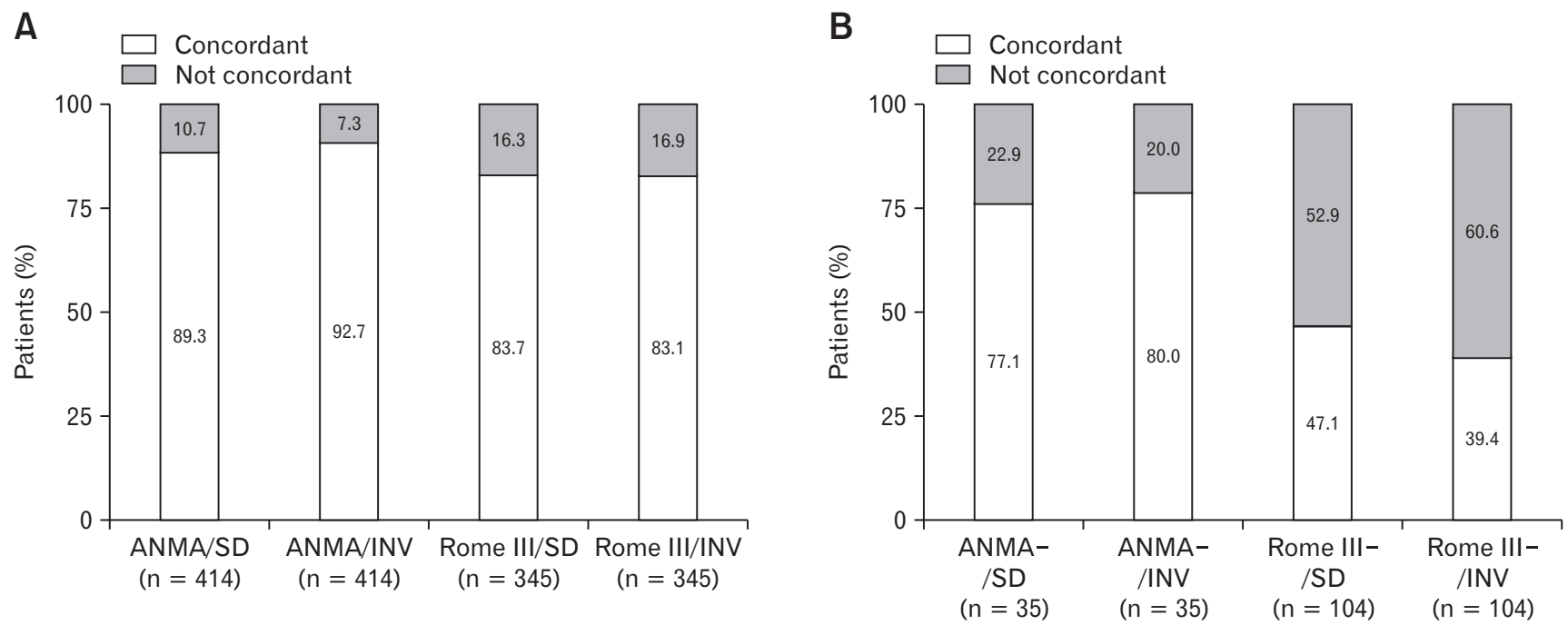

Figure 3. Concordance between Asian Neurogastroenterology and Motility Association (ANMA) or Rome III criteria and self-defined (SD) or investigator-defined (INV) diagnosis of chronic constipation in all patients (A) and ANMA-negative/Rome III-negative patients (B). Overall, mean scores were lower among subjects who were ANMA-negative/Rome III-negative (Wilcoxon 2-sample test, $P<0.0001$ ).

3B). Therefore, a large proportion of Rome III- subjects had a positive diagnosis of $\mathrm{CC}$ according to the $\mathrm{SD}$ or INV criteria.

\section{Patient Assessment of Constipation}

\section{Symptoms scores (Patient Assessment of Constipation Symptom)}

Mean (standard deviation) total PAC-SYM scores were similar in Rome III+ and ANMA+ subjects (1.44 [0.61] vs 1.35 [0.63]) (Fig. 4A). PAC-SYM abdominal subscale scores were similar between $\mathrm{CC}$-positive and CC-negative subjects according to both the ANMA CC tool and Rome III criteria, whereas stool and rectal symptom scores were more than double in subjects with CC. Mean (standard deviation) stool symptom scores were, for Rome III+ vs Rome-: $1.96(0.82)$ vs $0.86(0.64)$ and for ANMA+ vs ANMA-: 1.81 (0.85) vs 0.43 (0.42). Mean (standard deviation) rectal symptom scores were, for Rome III+ vs Rome III- (Fig. 4A): 0.84 (0.84) vs 0.34 (0.55) and for ANMA+ vs ANMA-: $0.77(0.82)$ and $0.24(0.40)$.

\section{Previous Chronic Constipation Therapy}

Of the ANMA+ subjects who entered stage $2 \mathrm{~B}$ of the study, $271(65 \%)$ had received CC treatment in the three months before the study. Of these, $80 \%$ had used one or more pharmacological treatment. Seventy-six percent had used one or more non-pharmacological treatment, and $29 \%$ had used one or more alternative therapy. Of the subjects who used a pharmacological therapy, $26 \%$ only used pharmacological therapy, $48 \%$ combined it with a nonpharmacological therapy, and $24 \%$ combined it with a non-pharmacological therapy and an alternative therapy.

Of the subjects who used a pharmacological therapy, the most commonly used were osmotic laxatives (56\%), stimulant laxatives (35\%), prokinetics (23\%), and probiotics (20\%).

Of the subjects who used a non-pharmacological therapy, the most commonly used were increased fiber intake $(76 \%)$ and diet modification (75\%). Of subjects who used an alternative therapy, $94 \%$ used traditional or herbal medicines.

\section{Difference between investigator's and subject's judgment about chronic constipation diagnosis}

With regard to current symptoms and co-morbidities, disagreement was observed in only 7\% (33/449) cases where the investigator reported a positive response and the patient reported negative. Furthermore, only 4\% (20/449) cases were recorded where the investigator reported a negative response and the patient reported positive. However, for patients who thought they had CC but did not screen positively, the agreement with the investigator would probably be much lower.

\section{Quality of life scores (Patient Assessment of Constipation- Quality of Life)}

The PAC-QOL questionnaire was completed by 414 

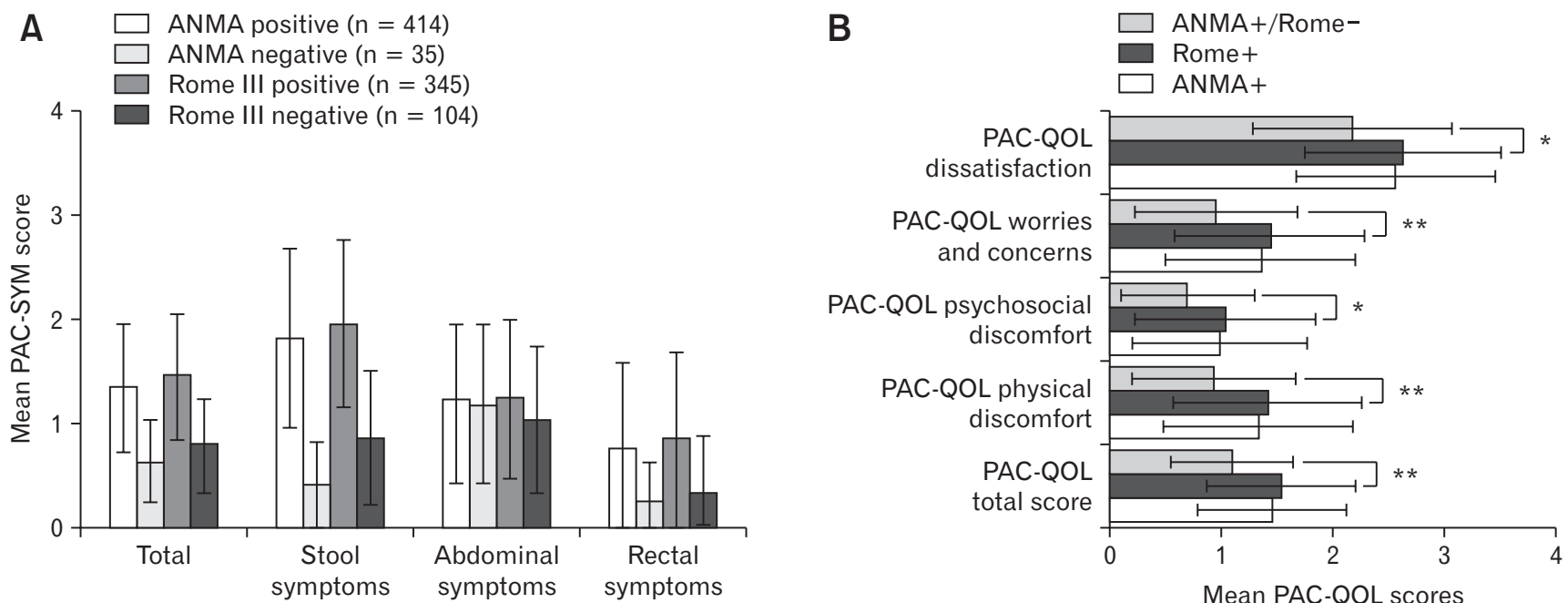

Figure 4. Symptom and quality of life (QOL) scores from the (A) Patient Assessment of Constipation Symptom (PAC-SYM) and (B) Patient Assessment of Constipation Quality of Life (PAC-QOL) ${ }^{\dagger}$ questionnaires. The items on both questionnaires were rated on a 5-point scale, with higher scores reflecting increasing severity of constipation-related symptoms (PAC-SYM) or greater impairment of QOL (PAC-QOL). In panel A, patients with chronic constipation (CC) had higher total, stool and rectal symptom scores compared to patients without CC. In panel B, QOL scores were similar in ANMA-positive and Rome III-positive patients. Levels of significance, determined by the Wilcoxon 2-sample test, are indicated by asterisks: ${ }^{*}<0.001 ;{ }^{*} P<0.0001$. PAC-SYM data are mean \pm standard deviation for all screened positive patients $(\mathrm{n}=449)$. PACQOL data are mean \pm standard deviation for all ANMA-positive patients $(\mathrm{n}=414)$.

ANMA+ subjects (Fig. 4B). Mean PAC-QOL total scores were similar in Rome III+ and ANMA+ subjects (1.53 [0.67] vs 1.45 [0.67]). Overall, mean scores were significantly lower among subjects who were ANMA+ but Rome III- (1.09 [0.55]; $\mathrm{n}=$ 69; $P<0.0001$, Wilcoxon-2-sample test).

\section{Discussion}

The ANMA criteria are based on the Rome III assessment, with the primary difference being a pre-diagnostic alert symptom checklist with the ANMA test. In all other regards the CC symptoms are identical between the ANMA and Rome III criteria; however, the minimum criteria for a positive diagnosis are lower with the ANMA test where one symptom and only 3 months' duration are required. The requirement for a single symptom is reinforced by the pre-diagnostic alert entry step, and the 3 month symptom duration is based on uncertainty with regards to the accuracy of recall beyond 3 months. These considerations are important when it is remembered that the ANMA criteria are intended for day-to-day clinical management within primary and secondary care, and not for academic research or for recruitment of patients for specialized treatments, such as biofeedback and surgery.

The primary objective of this study was to assess agreement/ disagreement between the ANMA CC tool and Rome III criteria (ie, the western "gold standard") for the diagnosis of CC in Asian subjects. The agreement between the Rome III criteria and the ANMA CC tool indicated that $76.8 \%$ of subjects had a Rome III+/ANMA + diagnosis and $7.8 \%$ had a Rome III-/ANMAdiagnosis, resulting in an overall agreement of $84.6 \%$ and disagreement of $15.4 \%$. These findings demonstrate that diagnosis of CC based on the ANMA CC tool agrees with that of Rome III criteria in the majority of Asian subjects with CC alert symptoms.

There are variations in the prevalence and symptoms of CC between East and West and also amongst countries within Asia which complicate the implementation of a standardized diagnostic approach. For instance, patients from Asia have a shorter colonic transit time than Western patients, and in South Korea most patients who experience hard stools do not meet Rome criteria for hard stools. ${ }^{5,9}$ There are also differences within Asia; in India, patients with self-perceived constipation report a median of 2 bowel movements per day ${ }^{10}$ and in China, where there are marked regional differences in the prevalence of constipation. ${ }^{11}$ Similarly, and as observed in our results, there are slight differences in the prevalence of $\mathrm{CC}$ among Malays, Indians, and Chinese in Singapore. ${ }^{12}$

A more reliable and accurate diagnosis of $\mathrm{CC}$ is required to aid in the optimal management of this disease. However, there is often 
disagreement between how patients and physicians define CC, with patients focusing on symptoms rather than stool frequency. ${ }^{4,13}$ This suggests that patients are currently underdiagnosed by existing criteria. In the current study, concordance was high for a positive diagnosis of CC based on the ANMA and Rome III criteria. This study also shows that a positive diagnosis with either tool is in concordance with a positive SD and INV diagnosis. The majority of ANMA- subjects were also SD- and INV-. In contrast, a high proportion of Rome III- subjects were $\mathrm{SD}+$ and/or INV+, suggesting that the Rome III criteria may have failed to detect a substantial proportion of subjects who were considered to have CC according to themselves or by an investigator. Therefore, these results indicate that the ANMA CC tool correlates more closely with both subject- and INV CC, compared with Rome III criteria.

Two factors, duration of symptom assessment and differences in diagnostic criteria, may explain the differences between the ANMA $\mathrm{CC}$ and Rome III populations, and how these correlate with SD and INV CC criteria. Firstly, the duration of symptom onset for diagnosis using the ANMA CC tool is 3 months, which is shorter than the 6-month duration for Rome III criteria $^{14}$ (ie, criteria fulfilled for the past 3 months with symptom onset $\geq 6$ months prior to diagnosis). To offset this potential difference, we used a 3-month observation period for all diagnostic methods to ensure that consistent symptom profiles were captured within each tool. It would also be of interest to determine the proportion of ANMA+ patients with symptoms for $<6$ months vs $\geq 6$ months; however, these data were not collected within the current protocol and thus further analysis is not possible. Secondly, the ANMA tool utilizes broader diagnostic criteria than the Rome III tool. As a result of these differences, there may be a potential for overlap between $\mathrm{CC}$ and constipation-subtype IBS (IBS-C) when using the ANMA CC tool, whereas the Rome III criteria may more clearly differentiate CC from IBS-C. However, opposing this theory, a recent study has highlighted the difficulty in distinguishing between CC and IBS-C using Rome III criteria. ${ }^{15-17}$ Furthermore, it has been suggested that both CC and IBS-C are part of the same condition, ${ }^{18}$ and similar therapeutic strategies are often utilized. Ultimately, further study is required to assess the differential diagnoses in those patients who are Rome III- but ANMA+.

CC prevalence rates reported in this study were variable between the different diagnostic methods and countries. The prevalence of CC in China and South Korea was higher when SD, compared with that of Singapore, where prevalence of SD CC appeared to have a much lower frequency than INV CC or a positive diagnosis according to the ANMA CC tool and Rome III criteria.
This may have been because patients from Singapore were recruited from a single center in a private healthcare institution comprising of primary and secondary care level patients. Garrigues et $\mathrm{al}^{19}{ }^{19}$ lso reported differences in the prevalence of $\mathrm{CC}$ according to selfreported, Rome I, and Rome II criteria. Differences in prevalence among Asian countries have also been previously reported using SD criteria. ${ }^{1}$ Furthermore, the higher prevalence rates of $\mathrm{CC}$ in female versus male subjects in the current study is consistent with those in North America, Europe, and Oceania. ${ }^{20,21}$

The symptoms of $\mathrm{CC}$ are unpleasant and have an adverse effect on patients' QoL. ${ }^{22,23}$ In the present study, PAC-QOL scores were similar between ANMA+ and Rome III+ subjects, but tended to be lower in ANMA+/Rome III- subjects suggesting that when defined by one tool only ie, less broad criteria, a difference may be more apparent. Therefore, consistent $\mathrm{QoL}$ findings were seen in subjects with agreement between the 2 diagnostic methods. An interesting observation was the very low symptom scores seen in patients who were ANMA-. This is consistent with the broad criteria used in the ANMA assessment and hence its ability to detect patients with very mild symptoms that may not be detected as CCpositive by the more stringent Rome III criteria. Therefore, the use of the ANMA CC tool offers the opportunity to detect patients with milder forms of $\mathrm{CC}$ and those in whom symptoms have not progressed to a severe stage. Furthermore, in a recent clinical trial of prucalopride, improvements in PAC-SYM and PAC-QOL subscales was associated with improved symptoms among Asian and non-Asian patients with CC. This was evident in patients in both active and placebo treatment arms. ${ }^{24}$

In conclusion, a high level of agreement was seen for the diagnosis of CC according to the ANMA CC tool and Rome III criteria in Asian patients with $\mathrm{CC}$ alert symptoms. Furthermore, the ANMA CC tool demonstrated higher agreement with both SD and INV criteria than Rome III criteria. Therefore, the ANMA $\mathrm{CC}$ tool appears to be more sensitive than Rome III criteria for diagnosing $\mathrm{CC}$ in Asia patients. Further prospective studies are warranted to confirm the applicability of the ANMA CC tool in a wider Asian population.

Acknowledgements: Medical writing and editorial assistance was provided by Maxwell Chang BSc Hons of ApotheCom, Singapore. This assistance was funded by Janssen Pharmaceutical Companies of Johnson \& Johnson.

Financial support: This study was supported by Janssen Pharmaceutical Companies of Johnson \& Johnson. 
Conflicts of interest: Kok-Ann Gwee has received research grants from Abbott Laboratories and Janssen Pharmaceuticals; speaking honoraria from Abbott Laboratories, Janssen Pharmaceuticals, Boehringer Ingelheim International $\mathrm{GmbH}$, and Menarini Asia-Pacific; and served on advisory board/consultant for Danone Research, Abbott Laboratories, Janssen Pharmaceuticals, and Menarini Asia-Pacific. Paul Bergmans, Bogdana Coudsy, Angelia Sim, and JinYong Kim are full-time employees of Janssen Pharmaceutical Companies of Johnson \& Johnson, and shareholders of Johnson \& Johnson. Minhu Chen has received speaking honoraria from Xian Janssen Pharmaceuticals, Abbott Pharmaceuticals Co. Ltd and Menarini Holding Co. Ltd. John A Pangilinan has served on the advisory panel for Sanofi-Aventis, Ferring Pharmaceuticals and JW Pharma. Huahong Wang has received research grant from Xian Janssen Pharmaceuticals. Stanislas Bruley des Varannes has received speaking honoraria from Janssen Pharmaceuticals and Abbott Laboratories, research support from Biocodex, and served on an advisory board and as a consultant for Alfa Wassermann.

Author contributions: Contributed to the design of the study: Bogdana Coudsy, JinYong Kim, Stanislas Bruley des Varannes, and Kok-Ann Gwee; acquisition, analysis, and interpretation of data: Bogdana Coudsy, Huahong Wang, Lin Lin, Minhu Chen, Nayoung Kim, Paul Bergmans, Angelia Sim, Stanislas Bruley des Varannes, Xiaohua Hou, Kok-Ann Gwee, and Khean-Lee Goh; and Review, revision, and approval of the final draft of the manuscript: Bogdana Coudsy, JinYong Kim, Nayoung Kim, John A Pangilinan, Stanislas Bruley des Varannes, Kok-Ann Gwee, and Khean-Lee Goh.

\section{References}

1. Wald A, Mueller-Lissner S, Kamm MA, et al. Survey of laxative use by adults with self-defined constipation in South America and Asia: a comparison of six countries. Aliment Pharmacol Ther 2010;31:274-284.

2. Probert CS, Emmett PM, Cripps HA, Heaton KW. Evidence for the ambiguity of the term constipation: The role of irritable bowel syndrome. Gut 1994;35:1455-1458.

3. Lee TH, Choi SC, Park MI, et al. Constipation misperception is associated with gender, marital status, treatment utilization and constipation symptoms experienced. J Neurogastroenterol Motil 2014;20:379-387.

4. Herz MJ, Kahan E, Zalevski S, Aframian R, Kuznitz D, Reichman S. Constipation: a different entity for patients and doctors. Fam Pract 1996;13:156-159.

5. Park JM, Choi MG, Cho YK, et al. Functional gastrointestinal disorders diagnosed by Rome III questionnaire in Korea. J Neurogastroenterol Motil 2011;17:279-286.
6. Gwee KA, Ghoshal UC, Gonlachanvit S, et al. Primary care management of chronic constipation in Asia: The ANMA chronic constipation tool. J Neurogastroenterol Motil 2013;19:149-160.

7. Frank L, Kleinman L, Farup C, Taylor L, Miner P Jr. Psychometric validation of a constipation symptom assessment questionnaire. Scand $\mathrm{J}$ Gastroenterol 1999;34:870-877.

8. Marquis P, De La Loge C, Dubois D, McDermott A, Chassany O. Development and validation of the patient assessment of constipation quality of life questionnaire. Scand J Gastroenterol 2005;40:540-551.

9. Gwee KA, Lu CL, Ghoshal UC. Epidemiology of irritable bowel syndrome in Asia: something old, something new, something borrowed. J Gastroenterol Hepatol 2009;24:1601-1607.

10. Tandon RK, Prasad N, Gupta MC, Tandon BN. Stool weights and transit time in North Indians. J Assoc Physicians India 1976;24:807-810.

11. Chu H, Zhong L, Li H, Zhang X, Zhang J, Hou X. Epidemiology characteristics of constipation for general population, pediatric population, and elderly population in china. Gastroenterol Res Pract 2014;2014:532734.

12. Gwee KA, Siah KT, Wong RK, Wee S, Wong ML, Png DJ. Prevalence of disturbed bowel functions and its association with disturbed bladder and sexual functions in the male population. J Gastroenterol Hepatol 2012;27:1738-1744.

13. Sandler RS, Drossman DA. Bowel habits in young adults not seeking health care. Dig Dis Sci 1987;32:841-845.

14. Drossman DA. The functional gastrointestinal disorders and the Rome III process. Gastroenterology 2006;130:1377-1390.

15. Wong RK, Palsson OS, Turner MJ, et al. Inability of the Rome III criteria to distinguish functional constipation from constipation-subtype irritable bowel syndrome. Am J Gastroenterol 2010;105:2228-2234.

16. Rey E, Balboa A, Mearin F. Chronic constipation, irritable bowel syndrome with constipation and constipation with pain/discomfort: similarities and differences. Am J Gastroenterol 2014;109:876-884.

17. Cremonini F, Lembo A. IBS with constipation, functional constipation, painful and non-painful constipation: e Pluribus...Plures? Am J Gastroenterol 2014;109:885-886.

18. Shekhar C, Monaghan PJ, Morris J, et al. Rome III functional constipation and irritable bowel syndrome with constipation are similar disorders within a spectrum of sensitization, regulated by serotonin. Gastroenterology 2013;145:749-757.

19. Garrigues V, Gálvez C, Ortiz V, Ponce M, Nos P, Ponce J. Prevalence of constipation: agreement among several criteria and evaluation of the diagnostic accuracy of qualifying symptoms and self-reported definition in a population-based survey in Spain. Am J Epidemiol 2004;159:520-526.

20. Higgins PD, Johanson JF. Epidemiology of constipation in North America: a systematic review. Am J Gastroenterol 2004;99:750-759.

21. Peppas G, Alexiou VG, Mourtzoukou E, Falagas ME. Epidemiology of constipation in Europe and Oceania: a systematic review. BMC Gastroenterol 2008;8:5.

22. Glia A, Lindberg G. Quality of life in patients with different types of functional constipation. Scand J Gastroenterol 1997;32:1083-1089.

23. Wald A, Scarpignato C, Kamm MA, et al. The burden of constipation on quality of life: results of a multinational survey. Aliment Pharmacol 
Ther 2007;26:227-236.

24. Ke M, Tack J, Quigley EM, et al. Effect of prucalopride in the treatment of chronic constipation in Asian and non-Asian women: a pooled analysis of 4 randomized, placebo-controlled studies. J Neurogastroenterol Motil 2014;20:458-468. 